\title{
A Method of Numerical EXTENDING OF PERIOdICAL MOTIONS OF HAMILTONIAN SYSTEMS WITH RESPECT TO PARAMETERS
}

\author{
S.R. Karimov and A.G. Sokolsky \\ Institute of Theoretical Astronomy, St. Petersburg, Russia
}

\section{ABSTRACT}

The method proposed is a generalization of the method described in [1]. It is represented in terms of the familtonian formalism with all the advantages arising from it. A complete presentation of the method is given in the paper [2].

\section{FORMULATION OF THE PROBLEM}

Consider an autonomous generalized conservative mechanical system with $J+1$ degree of freedom, depending on $K$ parameters, with the Hamiltonian $H(\bar{z}, \bar{p})$, where $\bar{z}=[\underline{\bar{x}}]$ $\bar{x}=\left(x_{1}, \ldots, x_{J+1}\right)^{T}$ are coordinates, $\bar{y}=\left(y_{1}, \ldots, y_{J+1}^{\bar{J}}\right)^{T}$ are impulses and $\vec{p}=\left(p_{1}, \ldots, p_{K}\right)^{T}$ are parameters, of the system. Yie assume that the Hamiltonian function $H$ smoothly (in particular,analytically) depends upon its variables in the domain of their change under consideration.

The set of differential equations with such a Hamiltonian has an integral $H=h$, where $h$ is energy constant.

Differential equations themselves have the form:

$$
\bar{z}=I H_{z}
$$

Here $I=\left|\begin{array}{rr}0 & E \\ -E & 0\end{array}\right|$ is a unit simplectic matrix, $H_{z}=\partial H / \partial \bar{z}$. 
Let at some fixed values of the parameters, $\bar{p}=\bar{p}$ be known, the initial value $\bar{Z}(0, \bar{p})$ for any periodic solution of the equations (1.1) having period $T(\bar{P})$ :

$$
\mathrm{Z}=\overline{\mathrm{Z}}(\mathrm{t}, \overline{\mathrm{P}}), \quad \overline{\mathrm{Z}}(\mathrm{t}, \overline{\mathrm{p}})=\overline{\mathrm{Z}}(\mathrm{t}+\mathbf{T}(\overline{\mathbf{P}}), \overline{\mathrm{P}})
$$

In addition, the functions (1.2) themselves may be determined by numerical integration of the equations (1.1) over the interval $t \in[0, T]$.

One formulates a problem of constructing (and then al so studying the properties) pertodic solutions being the analytical continuations (with respect to parameters) of the solution (1.2). It means that it is necessary to find such periodic solutions

$$
\bar{z}=\bar{z}(t, \bar{p}), \quad \bar{z}(t, \bar{p})=\bar{z}(t+T(\bar{p}), \bar{p})
$$

which satisfy the conditions of belonging to the family of periodic solutions, generated by the solution (1.2)

$$
\lim _{\bar{p} \rightarrow \bar{p}} \bar{z}(t, \bar{p})=\bar{Z}(t, P), \quad \lim _{\bar{p} \rightarrow \bar{p}} T(\bar{p})=T(\bar{p}),
$$

\section{LOCAL COORDINATES}

Let us introduce the notations:

$$
\bar{\pi}=\overline{\mathrm{p}}-\overline{\mathrm{p}}, \quad \bar{\zeta}=\overline{\mathbf{z}}-\overline{\mathrm{z}}
$$

We shall consider in (2.1) increments of the parameters $\pi$ and the local coordinates $\overline{\bar{\zeta}}$ as independent of small quantities of the same order of smallness and retain'in the Taylor expansions of the $r i g h t-h a n d$ sides of the differential equations the first order terms only. Then one obtains for $\bar{\zeta}$ the linear canonical differential equations with the Hamiltonian (superscript $\zeta$ in the familtonian is an index).

$$
\begin{aligned}
& \mathrm{H}^{\zeta}=\frac{1}{2} \bar{\zeta}^{-\mathrm{T}} \mathrm{H}_{\mathrm{zz}} \bar{\zeta}+\bar{\zeta}^{-\mathrm{T}_{\mathrm{z}}} \bar{\pi}, \\
& \mathrm{H}_{\mathrm{zZ}}=\partial^{2} \mathrm{H} /\left.\partial^{2} \overline{\mathrm{z}}\right|_{0}, \mathrm{H}_{\mathrm{zp}}=\partial^{2} \mathrm{H} /\left.\partial \overline{\mathrm{z}} \partial \overline{\mathrm{p}}\right|_{0},
\end{aligned}
$$

where subscript " $\underline{ }$ " implies that after differentiation a substitution $\bar{z}=\bar{z}, \bar{p}=\bar{p}$ is performed, i.e. it is set that $\bar{\zeta}=\overline{0}$, $\bar{\pi}=\overline{0}$. 
The system of differential equations with the Hamiltonian (2.2) is a non-autonomous one but it is admitting the integral obtained from the energy integral by retaining the first order terms

$$
\begin{aligned}
& \mathrm{H}_{\mathrm{z}}^{\mathrm{T}} \overline{\bar{\zeta}}+\mathrm{H}_{\mathrm{p}}^{\mathrm{T}} \bar{\pi}=\Delta \mathrm{h}=\text { const. } \\
& \mathrm{H}_{\mathrm{z}}=\partial \mathrm{H} /\left.\partial \overline{\mathrm{z}}\right|_{O^{\prime}}, \quad \mathrm{H}_{\mathrm{p}}=\partial \mathrm{H} /\left.\partial \overline{\mathrm{p}}\right|_{0} .
\end{aligned}
$$

Denote

$$
V(t)=|\dot{\overline{\mathrm{Z}}}(\mathrm{t})|=\sqrt{\dot{\overline{\mathrm{Z}}}(\mathrm{t}) \dot{\overline{\mathrm{Z}}}(\mathrm{t})} \text {, }
$$

i.e. $V$ is an absolute value of "the generalized" velocity on the trajectory in the phase space. Then $V(t) \neq 0$ over whole interval $t \in[0, \infty)$.

Consequently, for each point of the trajectory there exists a tangent in the phase space and one may associate with the solution under consideration $\bar{Z}(t)$ a mobile (accompanying) coordinate system one axis of which is directed along the vector of the generalized velocity $\overline{\mathrm{z}}(t)$ and the others are situated in the hyperplane normal to the trajectory in a given point $\bar{z}(t)$ at fixed $t$.

Let $S$ denote any matrix of transition to a new coordinate system and let its columns be reciprocal1y orthogonal vectors composing a canonical (simplectic) basis. Moreover, let matrix $S$ have the following composition:

$$
\begin{aligned}
& S=(R, \bar{s},-I R,-I \bar{s}), R=\left(\bar{s}_{1}, \ldots, \bar{s}_{J}\right), S_{R}=(R,-I R), \\
& \operatorname{dim} R=2(J+1) \times J, \quad S^{T} S=E, S^{T} I S=I,
\end{aligned}
$$

where $\bar{s}=\frac{1}{\bar{V}} \dot{\bar{Z}}$ is a unit vector of the tangent to the trajectory at a given point. It would be noted that from the equations of motion (1.1) it follows $-\mathrm{I} \overline{\mathrm{s}} \equiv 1 / \mathrm{V} \mathrm{H}_{\mathrm{Z}}=1 / \mathrm{V} \operatorname{grad} \mathrm{H}$. Which implies that the unit vector $-I \bar{s}$ is orthogonal to the hypersurface $H=h$ which is integral one.

Denoting by $\bar{w}$ a vector of local coordinates in the new system of the coordinates:

$\bar{w}=\left[\begin{array}{l}\bar{u} \\ \bar{v}\end{array}\right], \bar{u}=\left[\begin{array}{l}\bar{n}_{u} \\ m_{u}\end{array}\right], \bar{v}=\left[\begin{array}{l}\bar{n}_{v} \\ m_{v}\end{array}\right], \begin{aligned} & \operatorname{dim} \bar{n}_{u}=\operatorname{dim} \bar{n}_{v}=J, \\ & \operatorname{dim} m_{u}=\operatorname{dim} m_{v}=1,\end{aligned}$ 
where $\bar{u}=$ coordinates and $\bar{v}=$ impulses. From geometrical point of view it implies that $m_{u}$ is a displacement along the trajectory, $m_{v}$ is a displacement orthogonal to the hypersurface $H=h$ and $\bar{n}_{u}$ and $\bar{n}_{v}$ are displacements along the normals with respect to $u$ the trajectory on the tangent hypersurface. Therefore, we shall call the displacements $\bar{n}_{u}$ and $\bar{n}_{v}$ the normal, $m_{u}$ the tangential and $m_{v}$ the energetic ones. Connection between the old and new coordinates is given by formulas:

$$
\bar{\zeta}=S \bar{w}=R \bar{n}_{u}+\overline{s m}_{u}=I R \bar{n}_{v}-I \overline{s m}_{v}
$$

The integral (2.3) in the new variables will be written as follows:

$$
\mathrm{Vm}_{\mathrm{v}}+\mathrm{H}_{\mathrm{p}}^{\mathrm{T}} \overline{\mathrm{T}}=\Delta \mathrm{h}
$$

By virtue of canonical form of the transformation (2.8), the differential equations for $\bar{w}$ are canonical. We reduce the order of the system at the expense of the integral (2.9) excluding variable $\mathrm{m}_{\mathrm{v}}$. As a result of such reduction we obtain a canonical system of differential equations with $\mathrm{J}$ degrees of freedom and the Hamiltonian

$$
H^{n}=\frac{1}{2} \bar{n}^{T} H_{n} \bar{n}+\bar{n}^{-} h_{1} \bar{\pi}, \bar{n}=\left[\begin{array}{c}
\bar{n}_{u} \\
\bar{n}_{v}
\end{array}\right]
$$

and an additional differential equation

$$
\dot{\mathrm{m}}_{\mathrm{u}}=\frac{\dot{v}}{\mathrm{v}} \mathrm{m}_{\mathrm{u}}+\mathrm{h}_{\mathrm{n}}^{\mathrm{T}} \overline{\mathrm{n}}+\mathrm{h}_{2} \bar{\pi} \text {, }
$$

where

$$
\begin{array}{ll}
H_{n}=S_{R}^{T}\left(H_{z z} S_{R}+I \dot{S}_{R}\right), & h_{n}=S_{R}^{T} I\left(I H_{z z} I+H_{z z}\right) \bar{s}, \\
h_{1}=-\frac{1}{\bar{v}} h_{n} H_{p}^{T}+s_{R}^{T} H_{z p}, & h_{2}=\frac{1}{\bar{v}} \bar{s}^{T}\left(I H_{z z} I+H_{z z}\right) \bar{s} \cdot H_{p}^{T}+\bar{s}^{T} I H_{z p} .
\end{array}
$$

In (2.10)-(2.12) for uniformity we have additionally introduced the following notations: $p_{K+1}=h, \pi_{K+1}=\Delta h$, 
$\mathrm{H}_{\mathrm{K}+1}=-1, \mathrm{H}_{\mathrm{zp}}=\overline{\mathrm{K}+1}$ (infact we have introduced the energy constant into the vector of parameters, having increased its dimension by 1 , and thus obtained a possibility to consider non-isonergetic displacements).

Thus, we have obtained the equations possessing a remarkable property: The equations for normal variables (2.10) are independent of the tangential and energetic variables.

\section{PREDICTOR-CORRECTOR METHOD}

An algorithm for finding the solution (1.3) is realized in two stages. First (predictor) we find linear displacements with respect to increments of the parameters of the initial conditions and the period and then, (corrector), for taking into account the nonlinear character of influence of the parameter increments, we construct a convergent iteration procedure for finding the isoparametric corrections to the initial conditions and the period.

Now we present the predictor part of the method.

We introduce displacements $\bar{\zeta}$ (local coordinates) according to formulas (2.1) and then the normal, tangential and energetic displacements by formulas from section 2. As a result we derive equations (2.9)-(2.11). All the coefficients $n$. $m_{u}$ and $m_{v}$ in these equations have the period $T$. form

Friting the period $T^{*}$ of the desired solution in the

$$
T *=T+\tau \text {, }
$$

and assuming $\zeta, \tau$ and $\bar{\pi}$ to be quantities of the same order of smallness.

Then from conditions of periodicity of the solution (1.3) for normal and tangential displacements, we find the following boundary conditions:

$$
\begin{aligned}
\bar{n}(0) & =\bar{n}(T)+s_{R}^{T}(0) \Delta \bar{Z}, \\
m_{u}(0) & =m_{u}(T)+v(0) \tau+s^{-T}(0) \Delta \bar{Z}
\end{aligned}
$$

Here $\Delta \overline{\mathrm{Z}}=\overline{\mathrm{Z}}(\mathrm{T})-\overline{\mathrm{Z}}(0)$. This quantity at the predictor stage is equal to zero, but it will be a non-zero one at the corrector stage.

Making use of independence of the boundary value problems (2.10), (3.2) and (2.11), (3.3), their linearity as well as the fact that displacement $m_{u}(0)$ may be set equal to zero 
(since displacement along the orbit does not change it) one derives

$$
\begin{aligned}
& \bar{n}(0)=-[N(T)-E]^{-1}\left[\bar{n}_{p}(T)+s^{T}(0) \Delta \bar{Z}\right] \\
& \tau=-\frac{1}{V}\left[\bar{M}^{T}(T) \bar{n}(0)+m_{p}(T)+\bar{s}^{T}(0) \Delta \bar{Z}\right] .
\end{aligned}
$$

where quantities in the right-hand sides are evaluated by integrating the following Cauchy problems:

$$
\begin{aligned}
& \dot{\overline{\mathrm{z}}}=\mathbf{I} \mathbf{H}_{\mathrm{z}}, \quad \overline{\mathrm{z}}(0)=\overline{\mathrm{z}}(0, \overline{\mathrm{p}}), \\
& \dot{\mathrm{N}}=\mathrm{IH}_{\mathrm{n}} \mathrm{N}, \quad \mathrm{N}(0)=\mathrm{E}, \\
& \dot{\bar{M}}=\frac{\mathrm{V}}{\overline{\mathrm{V}}} \overline{\mathrm{M}}+\mathrm{N}^{\mathrm{T}} \mathrm{h}_{\mathrm{n}} \quad \overline{\mathrm{M}}(0)=\overline{0}, \\
& \dot{\bar{n}}_{p}=I\left(H_{n} \bar{n}_{p}+h_{1} \bar{\pi}\right), \quad \bar{n}_{p}(0)=\overline{0}, \\
& \dot{m}_{p}=\frac{\dot{v}}{\bar{v}} m_{p}+h_{n}^{T} \bar{n}_{p}+h_{2} \bar{\pi}, \quad m_{p}(0)=0 .
\end{aligned}
$$

Finally, from (2.9) one finds the initial value $m_{v}(0)$ for the energetic displacement and by formulas (2.8), $v(2.1)$, (3.1) the initial values of $\bar{z}(0)$ and the period $T^{*}$.

The derived solution, however will be periodic only approximately, 1.e. the differences $\bar{z}\left(T^{*}\right)-\bar{z}(0)$ will be nonzero but small quantities of the second order with respect to increments of the parameters $\bar{\pi}$. Moreover, the derived (due to predictor's work) initial values are not situated on the given integral surface $H=h *$.

For refining the initial conditions and the period one uses the corrector part of the procedure.

Let, now (1.2) be a non-periodic solution of the equations (1.1) but such that in its close vicinity in the phase space there exists the periodic solution (1.3) corresponding to the required values of the varying inner parameters and the constant of energy $h^{*}$. The goal is to find the motion (1.3) by assuming the motion (1.2) as the initial approximation.

Vie introduce local coordinates by formulas (2.1), (3.1) (vhere now $\mathrm{T}=$ aporoximate value of the period, $\mathrm{T}^{*}=$ sought for value under the same meanings of the parameters) and shall look for corrections to the initial conditions $\bar{\zeta}(0)$ and the period $t$ at $\bar{\pi} \equiv \overline{0}$, excepting $\Delta h=h^{*}-H(\bar{Z}(0), \bar{p})$.

By introducing the normal, tangential and energetic corrections by formulas (2.8) (where matrix $s(t)$ is constructed 
as "almost" periodical, 1.e. $S(T)-S(0)$ is a quantity of the same order as (2.1) one derives the equations (2.9)-(2.11).

Boundary conditions for these equations are derived from the requirement of $T *$ periodicity of the solution (1.3) and have the form $(3.2)-(3.3)$. In these formulas the quantity $\Delta \bar{z}$ is already a non-zero one but we consider that it is of the same order of smallness as $\tau$.

The solution of the obtained boundary value problems is sought in the same way as in predictor.

The relative error of the corrector work is defined by the quantity

$$
\varepsilon=|\Delta \overline{\mathrm{Z}}| / \max _{t \in[0, T]}|\overline{\mathrm{Z}}(\mathrm{t})|
$$

If the obtained quantity $\varepsilon$ turns out to be smaller than the initially prescribed quantity $\varepsilon^{*}$ then we regard the periodic motion (1.3) to be found. If the error of (3.4) is larger than the prescribed one then the corrector work should be repeated upto attainment of the inequality $\varepsilon<\varepsilon^{*}$. In addition, one naturally should, before the beginning of a new step of corrector's work, put $\bar{Z}(0)=\bar{z}(0), T=T^{*}$.

It would be noted that at each step of the corrector one computes corrections to the initial conditions and the period of the next order of smallness as compared to corrections of the previous step. Therefore, the constructed corrector possessess a quadratic convergence of the Newtonian type, clearly on condition that one successfully chooses the initial approximation generated by the predictor.

\section{REFERENCES}

[1] Karimov, S.B.,, Sokolsky, A.G. Periodic motions, generated by Lagrangian solutions of the circular restricted threebody problem// Celestial Mechanics, 1989, V.46, N.4,pp . 335 -381.

[2] Karimov, S.R., Sokolsky, A.G. A method of extending (with respect to the parameters) the natural families of periodic motions of Hamiltonian systems. - Preprint ITA Acad. Sci. USSR, 1990, N.9, $32 \mathrm{pp}$. 